\title{
STUDIES ON EXTERNAL GEOMETRY OF A PLANE WITH PHOTOGRAMMETRIC METHODS AND LASER SCANNING
}

\author{
S. Mikrut $^{1} *$, J. Brzęczek $^{2}$ \\ ${ }^{1}$ AGH University of Science and Technology, Faculty of Mining Surveying and Environmental Engineering, Al. Mickiewicza 30, \\ 30-059 Cracow, Poland - smikrut@agh.edu.pl \\ ${ }^{2}$ Rzeszow University of Technology, Faculty of Mechanics and Technology, ul. Powstańców Warszawy 12, 35-959 Rzeszów, \\ Poland - j.brzeczek@prz.edu.pl
}

Commission TCII

KEY WORDS: external geometry, photogrammetry, laser scanning, accuracy, UAV

\begin{abstract}
:
The authors of the paper were looking for a simple methods of the examination the airframe structure framework as part of the scientific and research project. The main tasks were: carrying out tests and analyses in defining external geometry of a selected plane with three independent methods i.e., geodetic (surveying), photogrammetric and laser scanning and selection of one of these methods as fast and acceptable accurate (below $3 \mathrm{~mm}$ ) measurement applied to examine plane geometry. The carried out experiments were mainly aimed at answering the question if tested measurement methods give satisfying and acceptable results in case of works referring to the objects such as plane and if the assumed accuracy is realistic to be achieved by these measurement techniques. The authors testing the available on the market methods of the measuring the geometry of planes in the aspect of the selection of most accurate, but at the same time, fast measurement method. Thus three independent methods were tested: classical geodetic measurement, laser scanning by means of two independent scanners and photogrammetric method (based on the generated cloud of points). Experiments carried out so far in various centres worldwide usually included measurements of single elements or small models of planes. In surveying measurements of large objects, mainly buildings are predominant (e.g. surveying or photogrammetry in the inventory of historic architectural monuments, studies of the geometry of engineering constructions, etc.). The objective of the experiment carried out by the authors was to find good solution somewhere in the middle, i.e., apply geodetic measurement methods, but apply them for so-called medium-size objects such as planes
\end{abstract}

\section{INTRODUCTION}

\subsection{Main task of the project}

The main tasks of the project were:

- carrying out tests and analyses in defining external geometry of a selected plane with three independent methods i.e., geodetic (surveying), photogrammetric and laser scanning; - selection of one of these methods as fast and accurate (below 3 $\mathrm{mm}$ ) measurement applied in defining the geometry of palne. In the framework of the project test measurements and a $3 \mathrm{D}$ model of aircraft EM-11C "Orka" were made and three independent methods were applied such as:

- classical geodetic measurement with a polar method,

- measurement method of laser scanning with two independent scanners,

- measurement with photogrammetric method (based on photogrammetric images: terrestrial method and drones).

The analysis of airframes and theirs elements geometrical accuracy and methods used during fabrication wasn't subject of the article

\subsection{Related work}

Present measurement techniques and methods of calculating and presenting the results provide wide range possibilities of applications. In literature there are also positions covering geometry of the whole objects (or their elements) depending on the accuracy and specific applications. This also takes place in the aviation, especially in operating conditions i.e. current and extraordinary inspections.

In literature we come across measurement methods generally accepted in solving various problems connected with studying the geometry of different elements with directly or indirectly methods connected with aviation industry.

For example, the publication (Rychlik et al., 2011), refers to the application of reverse engineering in $3 \mathrm{D}$ model with CAD techniques to carry out flatter studies. The authors applied an optical scanner of structural light of measurement resolution $0.05 \mathrm{~mm}$. Nevertheless, the measurement covered a relatively small object - a physical model of an plane $(3.3 \times 2.41 \mathrm{~m})$, and a numeric model was based on the network of triangles and NURBS area, based on skeleton curves and leading curves. As a result of works an error in the reconstruction of the geometry of an plane model was obtained on the level $+/-0.5 \mathrm{~mm}$ (authors did not give the confidence level, but the result was relatively good).

The studies on the geometry of selected elements are usually carried out with the application of so-called coordinate measuring machines - CMM. An example of the study of the geometry of the blade of the engine can be found in literature (Budzik et al., 2014). The authors studied the geometry applying two independent instruments i.e., ZEISS ACCURA and WENZEL LH 87 of type CMM. The applied by the authors

\footnotetext{
* Corresponding author
} 
software allowed later accuracy analyses of the measured element, referring to the nominal model. With such precise instruments the measurement accuracy ranges between 10 and 100 micrometers. The drawback of these instruments is that they can only make measurements for small-size objects (less than 10 metres).

In case of such small objects and such instruments the accuracy below $0.1 \mathrm{~mm}$ is expected. It was practically published in many papers. One of the authors of previously quoted publication in the previous paper (Budzik, 2010) carried out the studies of geometric accuracy of the models of the blades of aviation engines made with growth methods of fast prototype making FDM and SLA, obtaining the assumed accuracy.

More about the measurement techniques and coordinate measuring machines applied for small objects can be found in literature (Ratajczyk E., 2005) and (Ratajczyk E. et al, 2016). The authors also did their first work with some aspects of planes and published (Brzeczek et al., 2016). Procedures for the integration of photogrammetric and scanning data have been described in the literature based on experts led by the paper (Bęcek et al, 2015), (Głowienka et al., 2015). These techniques were used also e.g. for ship hull modelling (Burdziakowski et al., 2019) or for nondestructive testing of the miter gates (Binczyk et al., 2020). In the integration of scanning data, other contemporary mathematical models, such as wavelet ones, have also started to be used (Kędzierski et al, 2014), which is also an interesting approach and the authors of the publication plan to test these methods in the future. Plane geometry testing can have many applications. A lot of applications can be found in papers e.g. aerodynamic analysis, data fusion of variable fidelity data and handling quality analysis by flight simulation (Zhang et al, 2018). Measurement dedicated method used to examine of airframe and large parts geometry (including 3D method) during fabricate weren't analysed due to their useless in operating conditions.

\section{METHODOLOGY}

\subsection{Measurement methods.}

Based on literature one can notice several solutions in the measurement of medium-size objects, such as aircraft EM-11C, the object of our experiments. General breakdown of the applied methods is the following:

- classical direct measurement (the value of the measured dimension is directly obtained, without necessity of additional calculations based on functional dependence connected with the method or measuring instrument),

- geodetic optical measurement, widely applied earlier and now in aviation (also with the application of laser geodetic instruments) in case of larger dimensions,

- coordinate measuring techniques,

- optoelectronic measuring methods, which can be divided into active and passive.

The studies on the geometry of aircraft EM-11C "Orka" were prepared as three independent measuring experiments based on the same set of photo-points (reference points).

\subsection{Classical direct measurement.}

The idea of the direct geodetic classical measurement means precise measurement of the angles and distance to mark the spatial coordinates of selected points. In the framework of this task, the points were designed and more or less uniformly distributed on the surface of the aeroplane, earlier marked with black and white chequers glued to the surface of the aeroplane for the time of the measurement. It was a priori assumed that the geodetic method gives the accuracy on the level of $1 \mathrm{~mm}$, which was confirmed in the studies.

In case of classical direct geodetic measuring (also combined, such as levelling, carried out with the application of the methods of optical surveying and direct measuring) uncertainty of measurements (the most simplified form) can be generally expressed with the formula for MPE - Maximum Permissible Error (1), usually given by the producer of the instrument, e.g.:

$$
\mathrm{MPE}=+f-\left(\mathrm{P}+\frac{\mathrm{L}}{\mathrm{K}}\right)[\mathrm{\mu m}]
$$

where:

L - means the measured geometrical size ;

$\mathrm{K}$ - coefficient specified by instrument manufacturer;

$\mathrm{P}$ - coefficient specified by instrument manufacturer.

More details can be found in the literature (Bęcek et al, 2015), (Głowienka et al., 2015).

\subsection{Laser scanning method}

The errors of the measurements of scanners also include: instability of the speed of scanning (error difficult to compensate), errors of optical systems, errors of deflector and detector. In case of scanners the value of MPE (2) in the function of the measured dimension, given by the producer can be expressed by the formula considering maximal length parameter of the measured dimension 1 :

$$
M P E=+1-\left(x+\frac{L}{c}\right)[\mu m]
$$

where:

$\mathrm{L}$ - is the measured size;

$\mathrm{X}$ - characteristic geometrical dimension (length, dimeter, etc.) should be consulted with the manufacturer;

$\mathrm{C}$ - value defined by the manufacturer of the instrument.

In the carried out experiment, the measurement method of laser scanning involved scanning the aeroplane with two independent scanners, i.e., FARO FOCUS 3D and SURPHASER of selected checkpoints. Before starting with the scanning 15 reference zones were selected and 30 measurement shields were glued to plane. Reference points allowed automatic distribution of scans, which facilitated the process of cameral processing. Laser scanning method provides data in the form of the cloud of points (i.e., points of known coordinates $\mathrm{X}, \mathrm{Y}, \mathrm{Z}$ and known intensity of reflection), joint in one large file. Additionally, if there is a possibility to take digital images, the cloud of points can be „colored”, which gives a new product and has the advantage that a object 3D can be visualized in natural colours. More details can be found in the literature (Bęcek et al, 2015), (Głowienka et al., 2015).

\subsection{Photogrammetric method}

This method involved taking photogrammetric images from an aeroplane. The photos were taken as convergent for all the elements of the plane and stereograms for the selected parts of the aeroplane.

For the photos taken as convergent, a 3D model was constructed as the effect of image matching from two or more photos. As the effect of the work a product similar to the cloud of points obtained from the scanning, but much denser (one can practically map pixel by pixel). Generated in such a way network of points makes a 3D model, from which selected elements of the studied object can be differentiated. 
The situation error of the point is a conventional parameter not having deep probabilistic justification and this is the „resultant” of strictly established mean errors equalized by coordinates (Wiśniewski, 2005). For the analysis of the measurement of defining the accuracy of the situation of the measured points also the concept of RMSE is introduced. It is used in mathematical statistics RMSE (3), which is the root of the expected value of the square of "the error", i.e. the difference between the estimator and the estimated value.

$$
\operatorname{RMSE}\left(\Theta_{\text {est }}\right)=\sqrt{\left(\Theta_{\text {est }}-\Theta\right)^{2}}
$$

where:

$$
\begin{aligned}
& \Theta \text { - estimated value; } \\
& \Theta_{\text {est }} \text { - estimator } \Theta .
\end{aligned}
$$

More details can be found in the literature (Bęcek et al, 2015), (Głowienka et al., 2015).

\section{DESCRIPTION OF THE EXPERIMENTS}

\subsection{Description of the subject of studies}

The studies were carried out in the Margański and Mysłowski Zakłady Lotnicze Sp. z o.o. facilities. The object of the studies was aircraft EM-11C "Orka". The general view of the aircraft with the nominal dimensions is presented in figure 1 .

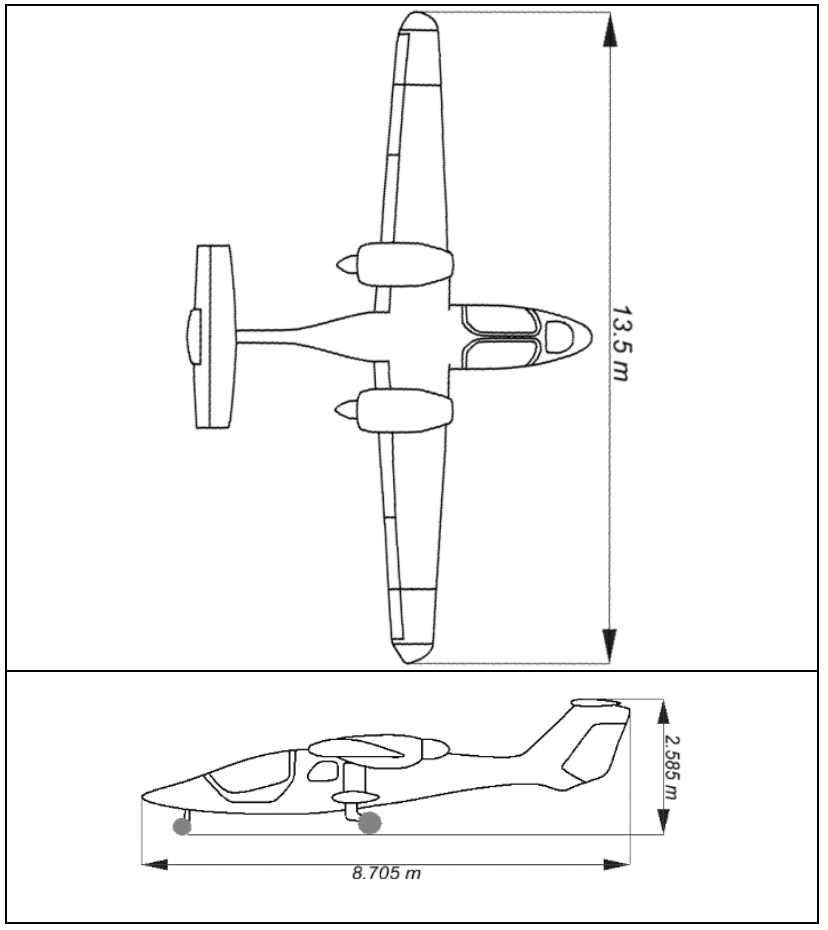

Figure 1 . The general view of the aircraft with the nominal dimensions.

\subsection{Measurements}

The measurement was carried out in the hangar by all the three methods based on the accepted of the system of coordinates:

- classical geodetic measurement,

- laser scanning measurement,
- photogrammetric measurement method (based on generated cloud of points from terrestrial camera carried by drone).

We start from experiments of classical geodetic measurement and laser scanning with two independent instruments i.e., Faro Focus 3D (distance accuracy $+/-2 \mathrm{~mm}$ on $10 \mathrm{~m}$ ) and Surphaser (distance accuracy $+/-0,7 \mathrm{~mm}$ on $15 \mathrm{~m}$ ). Then the obtained cloud of points was equalized and processed in programs Faro Scene and Leica Cyclone. Geodetic measurement was the measurement of coordinates $\mathrm{X}, \mathrm{Y}, \mathrm{Z}$ of points distributed in selected points of the plane, earlier signalled (Fig. 2). The measurement was carried out with the polar method, the accuracy was +/- $1 \mathrm{~mm}$. At the same time, there were independent photos taken with a non-metrical camera (Canon EOS5) and drone (Phantom-3 Professional" with camera Sony EXMOR 1/2.3), and the obtained cloud of points was equalized in program AgiSoft Photoscan. The obtained this method results were compared with each other one. The verification of the correctness of processing meant that the cloud of points generated with several independent methods was finally compared to the points measured by an independent geodetic method (based on verification coordinates obtained with the polar method). The scheme of the distribution of reference points is showed in figure 2 .

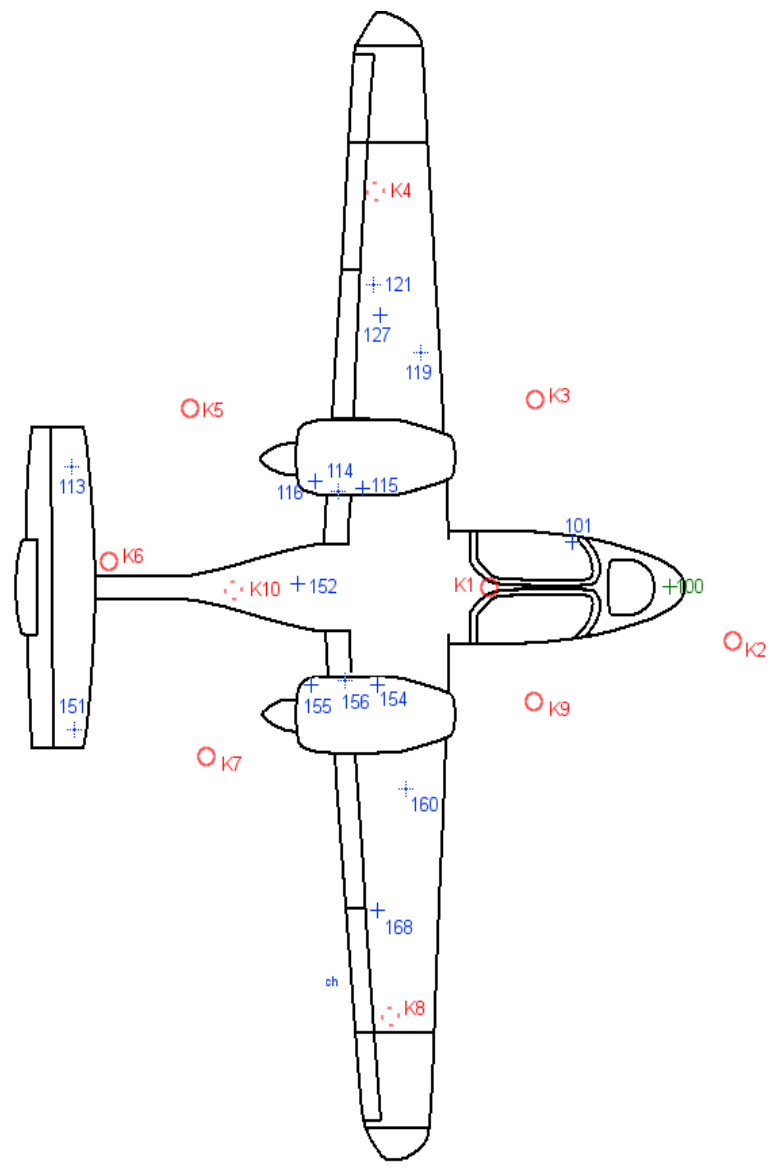

Fig. 2. The scheme of the distribution of reference points in the form of white spheres (in red), control points on the plane (in blue), control points used in the transformation of the cloud of points into the local system of surveying coordinates. 


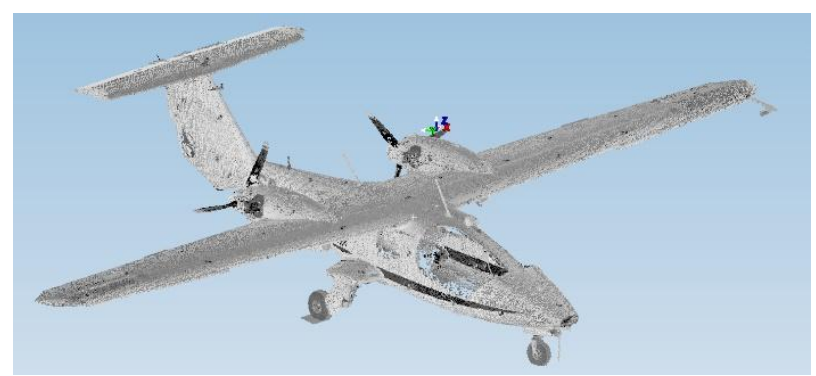

Figure 3. Effect of connecting and aligning data from individual stations in the adopted coordinate system

Thus the cloud models obtained with the method of laser scanning and photogrammetric method were transformed to the accepted local system of coordinates and then, based on visual identification, the points homological to the points measured with the geodetic method were localized. The visual effect was presented in figure 3, where all the laser measurements are put in one model 3D.

Table 1 contains the comparison of the accuracy for the subsequent scanners. This comparison shows that the instrument providing more accurate results is scanner Surphaser (despite the fact that during the measurement a smaller number of checkpoints was established, and consequently, the shorter time of the measurement).

\begin{tabular}{|l|c|c|}
\hline & Faro Focus 3D & Surphaser \\
\hline $\begin{array}{l}\text { Internal accuracy } \\
\text { of the matching } \\
\text { point clouds }\end{array}$ & $3,3 \mathrm{~mm}$ & $0,7 \mathrm{~mm}$ \\
\hline $\begin{array}{l}\text { Accuracy of the } \\
\text { transform point } \\
\text { clouds to control } \\
\text { points }\end{array}$ & $1,2 \mathrm{~mm}$ & $1,0 \mathrm{~mm}$ \\
\hline $\begin{array}{l}\text { RMS on control } \\
\text { points }\end{array}$ & $3,6 \mathrm{~mm}$ & $2,4 \mathrm{~mm}$ \\
\hline
\end{tabular}

Table 1. Set of accuracy for both scanners

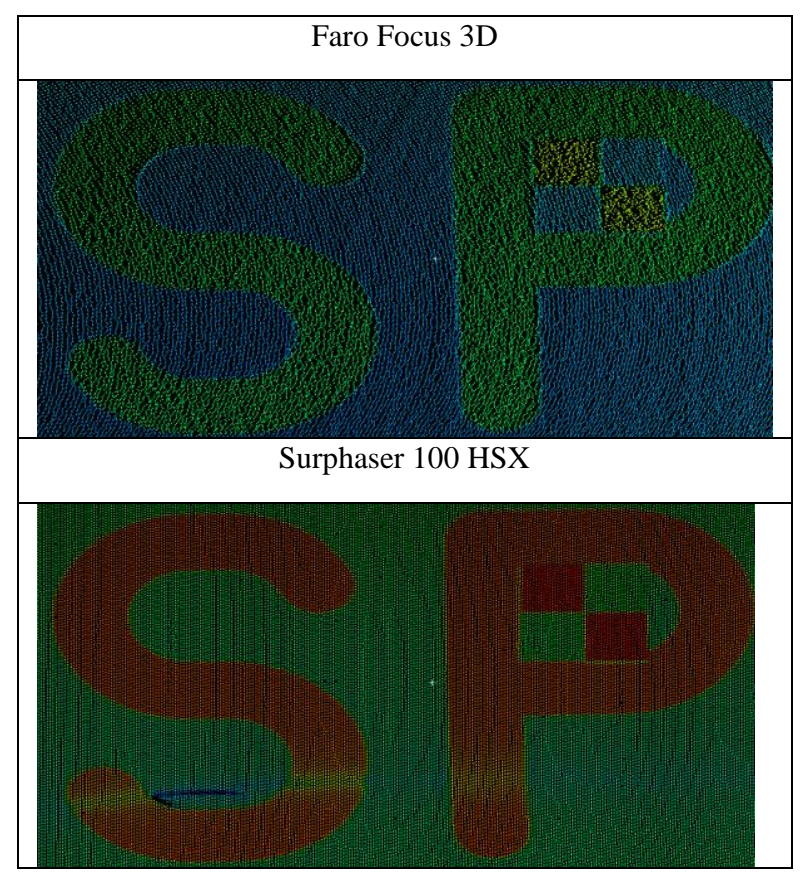

Figure 4. Reflection of points from the surface of the inscription SP.
Although the accuracy of the transformation of the clouds of points is on similar accuracy level (difference of $0.2 \mathrm{~mm}$ ), is a mean error on the controlled points, such as the mean error of the combining of the cloud of points is clearly lower for the mentioned instrument. This means that the method of laser scanning allows obtaining the results with the assumed accuracy below $3 \mathrm{~mm}$ (mean error on the controlled points for scanner Surphasr $2.4 \mathrm{~mm})$.

The advantage of scanner Surphaser over Faro Focus 3D is also visible during the analysis of the reflection of points from the surface of the plane. It is clearly visible that smooth surface of the plane is more precisely reflected by the scanning with scanner Surphaser (figure 4). Blue background and green letters after the scanning with scanner Faro have clearly higher standard deviation than their equivalents on the right had side in figures 5 .

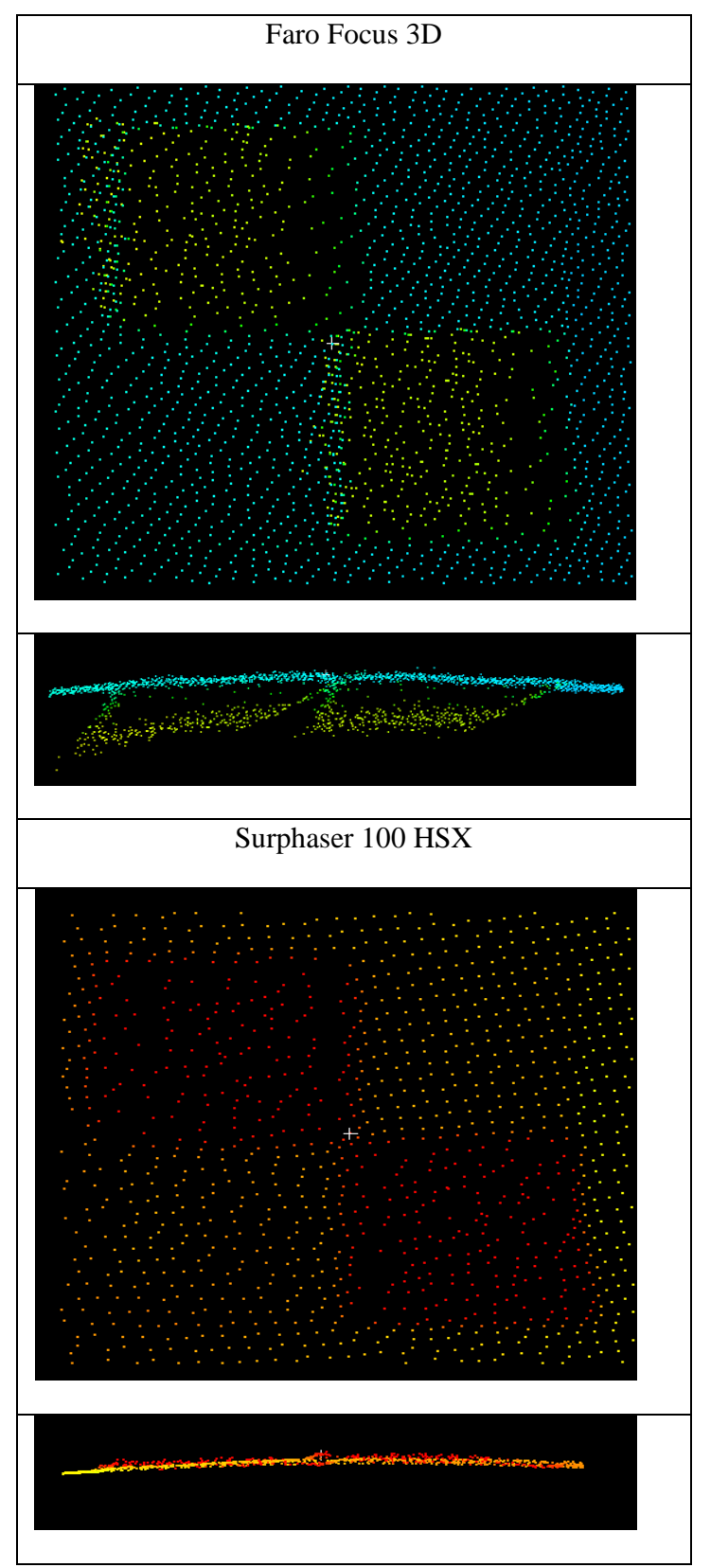

Figure 5. Comparison of the quality of reflection of points from the surface for both scanners(left) and section (right) 
In the photogrammetric measurement with this method, with the series of photos taken from terrestrial stands, the additional photos were taken with the use of the drone "Phantom Pro 3" (figure 6). The cloud of points generated from terrestrial photos was integrated with the photos taken with the method "from above". Mean error of adjustment into geodetic points did not exceed $3 \mathrm{~mm}$, which gives the accuracy on the level of laser measurement.

\subsection{Results}

Comparison of the measurement methods used for plane is presented in table 2 (RMS was computed after measurements). The obtained accuracy results indicate the geodetic method as most accurate $(+/-1 \mathrm{~mm}$ at $\sigma=1)$, however the disadvantage of this method is a long time of measuring.

\begin{tabular}{|c|l|l|c|}
\hline & Advantage & Disadvantage & RMS \\
[mm]
\end{tabular}

Table 2. The comparison of the methods

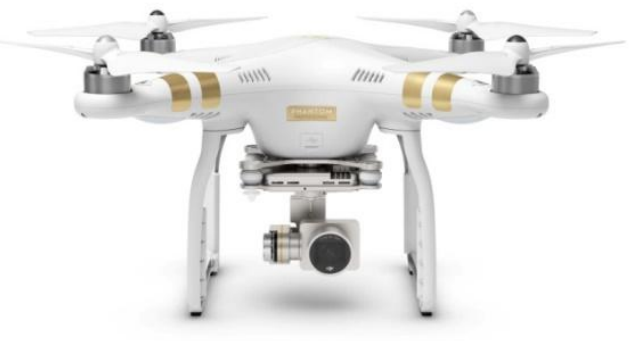

Figure 6. The drone "Phantom-3 Professional" with camera Sony EXMOR 1/2.3

The carried out experiments suggest that the most efficient method fulfilling the assumed tasks (accurate and fast) of the geometrical description of the object such as aircraft can be photogrammetric method, due to fast field measurement, but it should be remembered that the accuracy of field results in this method very much depends on a correct distribution of photos.

The lacks in the cover of images can result in uneven distribution, or even the gaps in the distribution of the cloud of points on the model, which can diminish the accuracy of the results.

\section{SUMMARY}

The objective of the studies was testing the available on the market methods of the measuring the geometry of planes in the aspect of the selection of most accurate, but at the same time, fast measurement method. Thus three independent methods were tested i.e.:

- classical geodetic measurement;

- laser scanning;

- photogrammetric method (based on the generated cloud of points).

The experiments showed that geodetic measurement turned out to be the most accurate (mean point situation error was about 1 mm - RMS). However, this method required the advanced instruments and an experienced observer to handle it. The measurement lasts several hours and requires almost ideal conditions i.e., windless weather. The limitation of this method is also the measurement of selected points only, thus when the measurement is ended, there is no possibility to add new points for the measurement

The measurement of the model of the method of laser scanning is characterized by the measurement on the accuracy level of 2$3 \mathrm{~mm}$ (depending on the equipment used), and the measurement requires the scanning of several measurement checkpoints and signalling reference points.

The time needed for signalling and measurement means 6-8 hours of work in the field, but the advantage of this method is obtaining the full 3D model, thus in any moment one can obtain any information on any point on the object with the accuracy of 2-3 $\mathrm{mm}$. This accuracy can still be elevated, but this requires additional efforts i.e., higher number of reference points and stands, which prolongs the measurement.

Third tested method involves making an large number of photogrammetric photos, and then - like in the case of laser scanning - generating of the cloud of points as an effect of the matching of photogrammetric photos. The obtained accuracy was on the level of the laser scanning accuracy (several $10 \mathrm{~mm}$ ). This method has a basic advantage: fast measurement in the field. Taking a series of photos, although in a large number, because above 500 , but the time necessary for taking them is 
only about 1 hour and any person, if properly instructed, can take them. This method is relatively novel and thus, requires further tests to elaborate effective geometry of the network of photos. In the opinion of authors, at the increased efficiency of the network it is possible to increase the accuracy $(1-2 \mathrm{~mm})$. The present accuracy is also affected by the fact that the calibration of the camera is carried out by the software. Carrying out the calibration in the laboratory conditions can increase the accuracy. The authors of this report plan the calibration in laboratory conditions in the nearest future.

Presented results show that the photogrammetric method fulfils the assumptions, thus fast measurement in the field and the accuracy of the processing and proper software allows us to obtain for the objects of the medium size the results of the accuracy equalling the one obtained from the scanners. This method requires the accurate analysis in terms of the efficiency of the distribution network. As proved by the authors' experience and experiments, the accuracy can also be influenced by the selection of proper equipment for the measurements (scanner, camera). The authors also see further work opportunities and increase accuracy by using sub-pixel methods (Czechowicz et al, 2010; Brzęczek et al, 2016, ).

\section{ACKNOWLEDGEMENTS}

This work was financed by research grants: 16.16.150.545 Acknowledgements to company Margański and Mysłowski Zakłady Lotnicze Sp. z o.o. for making the aircraft EM-11C "Orka" available for testing and Agnieszka Moskal, Elżbieta Pastucha for help in obtaining and developing the data.

\section{REFERENCES}

Bęcek K. , Gawronek P., Kwoczyńska B., Matuła P., Michałowska K. ,Mikrut S., Mitka B., Piech I., Makuch M., 2015, Modelowanie i wizualizacja danych 3D na podstawie pomiarów fotogrametrycznych i skaningu laserowego. Wydawnictwa WSIE, Rzeszów.

Binczyk, M., Kalitowski, P., Szulwic, J., Tysiac, P., 2020, Nondestructive Testing of the Miter Gates Using Various Measurement Methods. Sensors, 20, 1749.

Burdziakowski, P., Tysiac, P., 2019, Combined Close Range Photogrammetry and Terrestrial Laser Scanning for Ship Hull Modelling. Geosciences, 9, 242.

Brzęczek J. Mikrut S., 2017, The use of photogrammetry for special flights tests. Eksploatacja i Niezawodność Maintenance and Reliability; 19 (4): 597-603

Budzik G., Kubiak K., Zaborniak M., Przeszłowski Ł., Dziubek T., 2014, Analysis of Dimential Accuracy of Blade of Aircraft Engine Using a Coocrdinate Measuring Machine., Journal of KONES Powertrain and Transport, Vol. 21, No. 2.

Budzik G., 2010, Geometric Accuracy of Aircraft Engine Blde Models Constructed By Means of The Generative Rapid prototyping Methods FDM and SLA. Advances in Manufacturing Science and Technology, Vol. 34, No. 1.

Czechowicz A.,, Gryboś P., Jachimski J., Mikrut S., Mikrut Z., Pawlik P., Tadeusiewicz R, 2010, Sieci neuronowe w procesach dopasowania zdjęć lotniczych. Wydawnictwa AGH. Kraków.
Głowienka E., Jankowicz B., Kwoczyńska B., Kuras P., Michałowska K., Mikrut S., Moskal A., Piech I., Strach M., Sroka J., 2015, Fotogrametria i skaning laserowy w modelowaniu 3D. Wydawnictwo WSIE Rzeszów.

Kędzierski M., Fryśkowska A., 2014, Terrestrial and aerial laser scanning data integration using wavelet analysis for the purpose of 3D building modeling. M Kedzierski, A FryskowskaSensors, 14, 12070-12092;

Ratajczyk E., 2005, Współrzędnościowa technika pomiarowa, Monografia. Wydawnictwo OWPW.

Ratajczyk E., Woźniak A., 2016, Współrzędnościowe systemy pomiarowe. Monografia. Wydawnictwo OWPW.

Rychlik M., Niezgódka M., Hausa H., Kotecki K., 2011, Opracowanie komputerowej geometrii CAD modelu samolotu do badań flatterowych $\mathrm{z}$ zastosowaniem technik reverse engineering. Prace Instytutu Lotnictwa 220, s. 125-134, Warszawa.

Wiśniewski Z., 2005, Rachunek Wyrównawczy w Geodezji (z przykładami). Wydawnictwo Uniwersytetu WarmińskoMazurskiego w Olsztynie.

Zhang M., Jungo A., Gastaldi A.A., Melin T., 2018, Aircraft Geometry and Meshing with Common Language Schema CPACS for Variable-Fidelity MDO Applications. Aerospace, 5, 47. 